\title{
LOCAL BIFURCATION THEORY FOR THERMOELASTIC BRAVAIS LATTICES
}

\author{
By
}

J.L. Ericksen

IMA Preprint Series \# 729

November 1990 


\title{
LOCAL BIFURCATION THEORY FOR THERMOELASTIC BRAVAIS LATTICES
}

\author{
By
}

\author{
J.L. Ericksen
}

IMA Preprint Series \# 729

November 1990 


\title{
LOCAL BIFURCATION THEORY FOR THERMOELASTIC BRAVAIS LATTICES
}

\author{
J. L. Ericksen* \\ Department of Aerospace Engineering and Mechanics \\ and School of Mathematics, \\ University of Minnesota \\ Minneapolis, MN 55455
}

\begin{abstract}
Some weak first order phase transitions encountered in shape-memory alloys seem to occur near bifurcations which are not actually observed. Being curious about the nature of these in particular, I began to think about the nature of all of the bifurcation patterns predicted by the theory of thermoelastic Bravais lattices. Presented here are local characterizations of the most likely patterns near a bifurcation.
\end{abstract}

\section{Introduction}

As is made evident by some of the papers collected in reference [1], for example, nonlinear thermoelasticity theory describing Bravais lattices is proving to be useful for analyzing neartransition behavior of alloys exhibiting shape-memory effects. These involve Martensitic transformations, commonly of first order. From observations such as are discussed in various places in reference [2], it seems typical of these transformations that, as one cools an unloaded crystal which is in the Austenitic phase at higher temperatures, some linear elastic modulus becomes unusually small, seeming to extrapolate to zero at a temperature slightly below that at which the crystal transforms to the Martensitic phase. While there is little experimental evidence to support it, there is some opinion that a similar effect is likely to occur as the Martensitic phase is warmed, to get near the transformation temperature.

Theoretical experience suggests that the vanishing of such moduli will be associated with some kind of bifurcation, probably involving equilibrium branches too unstable to be observable. This led me to be curious as to what might be deduced from theory about the natures of such bifurcation patterns. Briefly and roughly, as I interpret it, the general problem is to get a qualitative

* Member of the Research Group on Transitions and Defects in Ordered Materials. 
description of the bifurcation patterns most likely to occur in thermoelastic Bravais lattices. There is a global problem, involving one or more connected sets of equilibrium branches, with any number of bifurcations. For the most part, I'll be concerned only with the nature of bifurcation patterns near one bifurcation, the local problem. A few remarks about a more global problem are included in Section 10.

One the face of it, this seems to involve a large number of possibilities. Physically, equilibria can have any of the symmetries associated with the seven point groups which can be realized in Bravais lattices, which are described in the Appendix, and branches with different symmetries can meet at bifurcations. With each point group is associated some number of elastic moduli, giving different possible choices of that which vanishes at bifurcation. However, we will see that there are ways to reduce these to just six different mathematical problems which I found tractable, although I am not expert in bifurcation theory. Certainly some of these, possibly all, are treated in the literature on bifurcation theory, although I have not yet found references to all. However, I found it hard to make some points without giving some indication of how the analysis can be done, so I have done this. Mathematically, there are additional possibilities but, by an old criterion which is to be discussed and used, they are unlikely to be encountered.

My purpose is to elaborate these remarks. To keep this paper from being unreasonably long, I will omit some of the details in various analyses, but indicate the kind of reasoning which is used in getting the results.

There are some different aspects to this study. One is the reduction of the numerous possibilities to the aforementioned six problems. One needs to understand this in some detail, to know how to relate any particular physical possibility to one of the six mathematical problems. Then, one needs some analysis of each of the six and, as noted above, I'll sketch mine. Finally, I'll discuss a particular case which seems to represent a rather common occurrence, experimentally. 
Some of the elementary bifurcation theory for Bravais lattices is discussed by Ericksen [3], but the present study goes far beyond this, to give a fairly complete local theory of the generic kind.

\section{Basic formulation}

Here, the absolute temperature is considered to be uniform and to be a control variable, and we will consider only homogeneous configurations of our Bravais lattices. It is a rather routine matter to generalize the analysis to include the possibility that a uniform hydrostatic pressure is also a control variable, but I won't do so. In our analyses, some homogeneous configuration will be taken as a reference so, for the configurations considered, the right Cauchy-Green tensor $\mathbf{C}$ will be independent of position. According to thermoelasticity theory, the Helmholtz free energy density $\phi$ is a function of the form

$$
\phi=\hat{\phi}(\mathbf{C}, \theta)
$$

While there are physical reasons to be interested in cases where $\hat{\phi}$ exhibits rather mild singularities at bifurcations, I think it better to avoid these complications, for a first look. At least I want to avoid the rather common assumption of analyticity, and its implications relating to analytic continuation. Thus, in the neighborhoods considered, I'll assume more than is necessary, that $\hat{\phi}$ is a $C^{\infty}$ function: different weaker assumptions suffice for different analyses.

As is discussed by Ericksen [4], for example, versions of such theory appropriate for Bravais lattices involve some special features. Briefly, $\hat{\phi}$ is to be invariant under an infinite discrete group conjugate to $\mathrm{GL}(3, \mathbf{Z})$, the group of general linear transformations on the integers, in three dimensions. However, the restriction of $\hat{\phi}$ to a suitably chosen neighborhood of a particular configuration, with $\mathbf{C}=\mathbf{C}_{0}$, say, is invariant only under a finite subgroup, that which leaves $\mathbf{C}_{0}$ invariant. If one chooses this to be the reference so that $\mathbf{C}_{0}=1$, the subgroup is just the point group $\mathrm{P}_{0}$ associated with this particular configuration, and all other configurations in the neighborhood 
have point groups which are subgroups of it. If such a configuration is associated with a value $\mathrm{C}=\overline{\mathrm{C}}$, it will have a point group $\overline{\mathrm{P}}$ described as follows:

$$
\overline{\mathrm{P}}=\left\{\mathbf{Q} \in \mathrm{P}_{0} \mid \mathbf{Q}^{\mathrm{T}} \overline{\mathbf{C}} \mathbf{Q}=\overline{\mathbf{C}}\right\},
$$

it being understood that, with $\mathbf{C}_{0}=1$, any possible $\mathbf{Q}$ is an orthogonal transformation, one of those described in the Appendix.

For us, equilibria will mean unstressed configurations, values of $\mathbf{C}$ and $\theta$ satisfying

$$
\frac{\partial \phi}{\partial \mathbf{C}}=0
$$

If $\left(\mathbf{C}_{0}, \theta_{0}\right)$ is one, one can try to use the implicit function theorem to infer the existence of smooth equilibrium paths $\mathbf{C}=\hat{\mathbf{C}}(\theta)$ through it, on which (2.3) is satisfied. What is important for this are some properties of the fourth order tensor

$$
\mathcal{B}\left(\mathbf{C}_{0}, \theta_{0}\right)=\frac{\partial^{2} \hat{\phi}}{\partial \mathbf{C} \partial \mathbf{C}}\left(\mathbf{C}_{0}, \theta_{0}\right)
$$

or, what is equivalent, $a$, the tensor of linear elastic moduli. Since properties of the latter are more likely to be familiar to the reader, I'll use it. To get $a$ from $\mathcal{B}$, one changes the reference configuration to make $\mathrm{C}_{0}=\mathbf{1}$, then takes

$$
a=4 \mathcal{B}\left(1, \theta_{0}\right) .
$$

In a way, this is a bit awkward since, if one is evaluating $a$ on a smooth branch, $\mathbf{C}=\hat{\mathrm{C}}(\theta)$, one needs to continually change the reference configuration to make $\hat{\mathbf{C}}(\theta)=\mathbf{1}$, but this is what fits rather common practice.

As defined, $a$ can be interpreted in the usual way, as a symmetric linear transformation on the space of symmetric second-order tensors. This involves considering the latter as equipped with the inner product 


$$
\mathbf{A} \cdot \mathbf{B}=\operatorname{tr} \mathbf{A B},
$$

with $\mathbf{A}$ and $\mathbf{B}$ representing any pair of symmetric second order tensors. In this setting, $a$ then has real eigenvalues and eigenspaces with the usual orthogonality properties.

Now, from the view of the implicit function theorem, the trivial case occurs when $\operatorname{ker} a=\varnothing$,

in which case, one has the local existence and uniqueness of the aforementioned smooth branch. On such a branch, as long as (2.7) holds, it follows that the point group is the same as that at the (fixed) reference, $\mathrm{P}_{0}$, also that the values of $\hat{\mathrm{C}}(\theta)$ are restricted by the obvious interpretation of (2.2): one can read off the possible forms from the description by Truesdell [5, Ch. IX], for example. Also, $a$, evaluated at any point on such a branch, will be invariant under $\mathrm{P}_{0}$. So, these are the normal branches, commonly considered in the linear thermoelasticity theory, for example. In fact, such theories also presume that the eigenvalues of $a$ are positive.

Our interest is more in limit points of such branches, at which (2.6) fails to hold. By continuity, they will satisfy (2.3), and the point group at the limit point will either be or include as a subgroup that on any of the normal branches having it as a limit point. Said differently, these are the places where bifurcations can occur.

Now, the intent is not to try to find all of the mathematical possibilities, but to look for the most likely. To assess this, I'll use an old criterion, still commonly used by physicists, which I trust. One could use instead more modern versions of generic bifurcation theory, but this involves making some sticky decisions about what is the appropriate topology, etc., like those discussed by Man [6] in his discussion of Gibbs' phase rule. In any event, what I'll do is to consider things of interest, like $a$, evaluated on a normal branch, as functions of $\theta$. As a consequence of invariance, they will satisfy some equations, to be viewed as identities. Otherwise, the view is that one equation might well be satisfied at a particular value of $\theta$, but it is unlikely that two or more 
independent equations will there be satisfied. There is a physical idea which supplies some motivation for accepting such ideas, which is also covered in the interesting discussion by Man [6]. Briefly, it involves the idea that a material is described not by one constitutive equation, but by a collection of slightly different equations, an idea which appeals to me.

In particular, with a value of $a$ being invariant under one of the seven point groups, its eigenspaces will also have this property and, in some cases, this requires that some of the eigenvalues be equal. By considering spectral representations, one can show that, when the number of distinct eigenvalues is as large as it can be, the eigenspaces are irreducible. That is, they contain no proper subspaces which are invariant under the applicable point group. It might well happen that two which could be different do coincide at a particular value of $\theta$, on a branch, but this would count as an equation. Now, for $a$ to have a non-trivial kernel, one of its eigenvalues must vanish. By our criterion, it is then unlikely that two eigenvalues will be equal unless, as a consequence of invariance, the two must be equal. This provides us with one important conclusion, viz.

Most likely, when ker $a \neq \varnothing$, the eigenspaces of $a$, in particular ker $a$, are irreducible. (2.8)

In Section 10, I illustrate a subtlety associated with this. Briefly, it is not considered unlikely that the point group at a limit point of some branch might be larger than that on the branch, and, when this happens, it might require some coalescence of eigenvalues or other conditions not then to be considered as equations. Later, we will make use of some other consequences of our criterion, but this one will be used repeatedly. So, for any of the seven point groups, an eigenvalue associated with any of the irreducible subspaces might vanish, and we need to consider all such possibilities. One could envisage the possibility that the list might be enlarged, by some consideration of the nature of branches for which the point considered is a limit point but, as we shall see, this is a problem which takes care of itself. 


\section{General scheme for reducing problems}

Essentially, we follow common practice in bifurcation theory, with some accounting for the quirks induced by invariance. The reference configuration is considered to be fixed, at a place where $\operatorname{ker} a \neq \varnothing$, at some particular temperature $\theta_{0}$. So, by assumption,

$$
\operatorname{ker} a \neq \varnothing
$$

and, as is suggested by (2.7), we also assume that the eigenspaces of $a$ are irreducible. Then, $\operatorname{ker} a$ is an irreducible space of some dimension $N$. Later, it will become clear that $\mathrm{N} \leq 3$. As a basis for symmetric second-order tensors, we introduce an orthonormal basis of eigenvectors of $a$, denoted by $\mathbf{E}_{\mathrm{i}}(\mathrm{i}=1, \ldots, 6)$,

$$
\mathbf{E}_{\mathrm{i}} \cdot \mathbf{E}_{\mathrm{j}}=\delta_{\mathrm{ij}}(\mathrm{i}, \mathrm{j}=1, \ldots, 6),
$$

with $\mathbf{E}_{1}, \ldots, \mathbf{E}_{\mathrm{N}}$ spanning ker $a$. In particular, values of $\mathbf{C}$ are represented by components $\mathrm{x}_{\mathrm{i}}$ as indicated by

$$
\mathbf{C}=\mathbf{1}+\sum_{\mathrm{i}=1}^{6} \mathrm{x}_{\mathrm{i}} \mathrm{E}_{\mathrm{i}},
$$

with $\mathrm{x}_{\mathrm{i}}=0$ at the putative bifurcation point. By the implicit function theorem, we can solve (2.3) locally to get relations of the form

$$
x_{j}=f_{j}\left(x_{1}, \ldots, x_{N}, \theta\right), j=N+1, \ldots, 6 \text {, }
$$

these functions being unique and smooth: they also satisfy the conditions

$$
\mathrm{f}_{\mathrm{j}}\left(0, \ldots, 0, \theta_{0}\right)=0
$$

Substituting these into the function $\hat{\phi}$, we get the reduced potential, represented by a smooth function of the form

$$
\Phi\left(\mathrm{x}_{1}, \ldots, \mathrm{x}_{\mathrm{N}}, \theta\right)
$$


Then, by standard reasoning, the remaining equilibrium equations reduce to

$$
\frac{\partial \Phi}{\partial x_{i}}=0
$$

and

$$
\frac{\partial \Phi}{\partial x_{i}}=\frac{\partial^{2} \Phi}{\partial x_{i} \partial x_{j}}=0 \quad \text { at } x_{i}=0, \theta=\theta_{0}
$$

where the indices take on the obvious $\mathrm{N}$ values. As I interpret the jargon used by physicists, $\mathrm{x}_{1} \ldots, \mathrm{x}_{\mathrm{N}}$ are "order parameters."

From remarks made earlier, $\hat{\phi}$ can be considered to be invariant under the point group $\mathrm{P}_{0}$ associated with the reference configuration and, from this and the aforementioned uniqueness of solutions, $\Phi$ inherits some invariance, which needs to be determined. The relevant point group involves some orthogonal transformations $\mathbf{Q}$, noted in the Appendix, transforming $\mathbf{C}$ to

$$
\mathrm{Q}^{\mathrm{T}} \mathrm{CQ}=\mathbf{1}+\sum_{\mathrm{i}=1}^{6} \mathrm{x}_{\mathrm{i}}\left(\mathrm{Q}^{\mathrm{T}} \mathbf{E}_{\mathrm{i}} \mathbf{Q}\right),
$$

and with $E_{i}$ as a basis, we can determine numbers $\lambda_{1}^{j}$ such that

$$
\mathbf{Q}^{\mathrm{T}} \mathbf{E}_{\mathrm{i}} \mathbf{Q}=\sum_{\mathrm{j}=1}^{6} \lambda \operatorname{li}_{\mathrm{i}}^{\mathrm{j}} \mathbf{E}_{\mathrm{i}}
$$

Of course, this induces on $x_{i}$ the transformation to the components $\bar{x}_{i}$ of $Q^{T} C Q$ given by

$$
\bar{x}_{i}=\sum_{i=1}^{6} \lambda x_{1} x_{j}, \quad i, j=1, \ldots, 6 .
$$

Now, since the inner product (2.5) is invariant under all orthogonal transformations, the $\mathrm{Q}^{\mathrm{T}} \mathrm{E}_{\mathrm{i}} \mathrm{Q}$ also form an orthonormal basis, whence follows that

$$
\wedge=\left\|\lambda_{1}^{j}\right\|, \quad i, j=1, \ldots, 6
$$


is an orthogonal matrix, of a rather special kind. That is, since the eigenspaces of $a$ are invariant under $\mathrm{P}_{0}, \wedge$ must map any such eigenspace onto itself. Thus, the obvious restriction of this linear transformation to an eigenspace is described by a matrix which is also orthogonal. In particular, this is true of the submatrix

$$
\lambda=\left\|\lambda_{1}^{j}\right\|, \quad i, j=1, \ldots, N
$$

describing the transformation of order parameters, so it satisfies

$$
\lambda^{-1}=\lambda^{T} \text {. }
$$

Also, it is easy to show that the matrices thus obtained form a group under matrix multiplication, which I'll call $\Re\left(\mathrm{P}_{0}\right)$, the reduction of $\mathrm{P}_{0}$,

$$
\lambda \in \mathfrak{R}\left(\mathrm{P}_{0}\right),
$$

Bear in mind that it depends on which eigenspace is considered to be ker $a$, as well as $\mathrm{P}_{0}$. To simplify notation a bit, we now write

$$
\mathbf{y}=\left(\mathrm{x}_{1}, \ldots, \mathrm{x}_{\mathrm{N}}\right) \text {. }
$$

After a bit of calculation, using the uniqueness of the solutions $(3.4)$, one finds that $\Re\left(\mathrm{P}_{0}\right)$ is the invariance group for the reduced potential, so

$$
\Phi(\lambda \mathbf{y}, \theta)=\Phi(\mathbf{y}, \theta), \quad \lambda \in \Re\left(\mathrm{P}_{0}\right)
$$

at least for $\mathbf{y}$ near the origin. Here, I have glossed one point, in that (3.17) makes no sense unless the domain of $\Phi$ is invariant under $\mathfrak{R}\left(\mathrm{P}_{0}\right)$. One can take care of this by considering the domain to be a suitably chosen neighborhood.

Another item of interest is the point group associated with $\mathbf{y}_{0}$, a particular value of $\mathbf{y}$ near the origin. To determine this, one calculates the subgroup $\mathfrak{R}\left(\mathrm{P}_{0}, \mathbf{y}_{0}\right)$ leaving $\mathbf{y}_{0}$ invariant:

$$
\Re\left(\mathrm{P}_{0}, \mathbf{y}_{0}\right)=\left\{\lambda \in \Re\left(\mathrm{P}_{0}\right) \mid \lambda \mathbf{y}_{0}=\mathbf{y}_{0}\right\}
$$


With (3.4), $\mathbf{y}_{0}$ determines a point $\mathbf{x}_{0}$ in the six-dimensional space $\left(\mathrm{x}_{1}, \ldots, \mathrm{x}_{6}\right)$, and $\lambda$ is a submatrix of at least one $6 \times 6$ matrix $\wedge(\lambda)$. From the uniqueness of solutions of (3.4), it then follows that

$$
\wedge(\lambda) \mathbf{x}_{0}=\mathbf{x}_{0}
$$

In turn, $\wedge(\lambda)$ is associated with at least one $Q \in P_{0}$, by (3.11); call it $Q(\lambda)$. From (3.3), $\mathbf{x}_{0}$ determines some value $\mathrm{C}_{0}$ of $\mathrm{C}$ and, with (3.19), we have

$$
\mathbf{Q}(\lambda)^{\mathrm{T}} \mathrm{C}_{0} \mathrm{Q}(\lambda)=\mathrm{C}_{0}
$$

The linear transformations $Q(\lambda)$ thus obtained then form some subgroup of $\mathrm{P}_{0}$ which, as is indicated in (2.2), is the point group associated with this particular configuration. From such theory it follows that the order of this group can be calculated by dividing the order of $\mathrm{P}_{0}$ by the number of distinct points in the orbit of $\mathrm{C}_{0}$ under $\mathrm{P}_{0}$ or, what is the same, the number of distinct points in the orbit of $\mathbf{y}_{0}$ under $\mathfrak{R}\left(\mathrm{P}_{0}\right)$. From the Appendix, it is clear that the order uniquely determines the point group. Frequently, I will use this idea to identify point groups.

Concerning the function $\hat{\phi}$, we have assumed that it is smooth, has some invariance locally, and admits some equilibria compatible with bifurcation. What is left rather tacit above is the notion that, at $\theta=\theta_{0}, \mathbf{y}=0$ represents a limit point of one or more normal branches: as it will turn out, it isn't important to make this more explicit. Otherwise, one can show that, for our local analyses, $\Phi$ need not satisfy any conditions beyond those mentioned explicitly above. Of course, any additional assumptions made about $\hat{\phi}$ could lead to additional restrictions on $\Phi$, not accounted for here.

To proceed with analysis of possible bifurcations, one needs to know the possible values of $\mathrm{N}$ and the groups $\mathfrak{R}\left(\mathrm{P}_{0}\right)$. Different physical problems can and do lead to the same $\mathrm{N}$ and $\mathfrak{R}\left(\mathrm{P}_{0}\right)$ although, in some cases, one needs to make some judicious choices of the eigenvectors, to make 
such identifications. So, this is the next step to be taken, to construct guide maps to link each of the physical possibilities to one of the mathematical bifurcation problems.

\section{Guide maps}

Essentially, this section is a tabulation of important information for each of the seven point groups described in the Appendix. It includes characterizations of the possible orthogonal irreducible subspaces which are, for our purposes, the possible eigenspaces. Also, where needed, I'll describe my special choice of the eigenvectors $\mathbf{E}_{1}, \ldots, \mathbf{E}_{\mathbf{N}}$, one which can be used to get the particular representations of $\mathfrak{R}\left(\mathrm{P}_{0}\right)$ noted below.

I'll not discuss derivations in detail, but some general comments might be helpful. One can start with the second-order tensors which are left invariant under the group $\mathrm{P}_{0}$, which are described by Truesdell [5, Chapter IX], for example. These form a linear space of some dimension. Associated with this are one-dimensional irreducible spaces, equal in number to the aforementioned dimension. If the number exceeds one, the particular form of such eigenvectors can be expected to be different for different materials, etc., for a given point group. This space will always include the identity, so all tensors in the orthogonal complement will be traceless. In this complement, there can be some one-dimensional irreducible spaces. If so, they will consist of tensors transforming to themselves by some elements of the group, to their negatives by other, making it easy to locate these.

If ker $a_{\text {is of the first kind, one has }}$

$$
\mathrm{N}=1, \quad \Re\left(\mathrm{P}_{0}\right)=\mathrm{SO}(1),
$$

where $\mathrm{SO}(1)$ is the proper orthogonal group in one dimension, the trivial group consisting only of the identity

$$
\mathrm{SO}(1)=1 .
$$

If $\operatorname{ker} a_{\text {is of the second kind, }}$ 


$$
\mathrm{N}=1, \quad \mathfrak{R}\left(\mathrm{P}_{0}\right)=\mathrm{O}(1)
$$

where $\mathrm{O}(1)$, the one-dimensional orthogonal group, also includes reflections

$$
\mathrm{O}(1)=1, \quad \mathrm{y}_{1} \rightarrow-\mathrm{y}_{1}
$$

Having determined all of these, and the space which they span, one can look in the remaining orthogonal complement for two-dimensional irreducible subspaces. It turns out that the possibility that one of these is ker $\boldsymbol{a}_{\text {leads }}$ to cases of the following kind

$$
N=2, \quad \Re\left(P_{0}\right)=G(\psi) ; \quad \psi=\pi / 2, \pi / 3 \text { or } 2 \pi / 3 \text {. }
$$

where $\mathrm{G}(\psi)$ is the group generated by the two matrices

$$
\left(\begin{array}{cc}
-1 & 0 \\
0 & 1
\end{array}\right),\left(\begin{array}{cc}
\cos \psi & \sin \psi \\
-\sin \psi & \cos \psi
\end{array}\right) .
$$

Having determined the two-dimensional possibilities, one can again take the orthogonal complement, and look for the three-dimensional possibilities. It turns out that there is just one of these, associated with cubic symmetry, and none of higher dimension. For it,

$$
\Re\left(P_{0}\right)=G(3),
$$

where $G(3)$ is a group of order 24 , generated by two improper orthogonal transformations $Q_{1}$ and $\mathrm{Q}_{2}$ which, in notation used in the Appendix, can be taken as

$$
\mathbf{Q}_{1}=-\mathbf{R}\left(\pi / 2, \mathbf{e}_{1}\right) \text { and } \mathbf{Q}_{2}=-\mathbf{R}\left(\pi / 2, \mathbf{e}_{2}\right) \text {. }
$$

With the point groups numbered as in the Appendix, I now tabulate important details. Tensors are described in terms of components, relative to the bases there used. 
No. 1

The irreducible subspaces are all one-dimensional, and consist of tensors left invariant under the group, orthogonal to each other, so (4.1) applies to any of them.

No. 2

Cases to which (4.1) applies involve four orthogonal one-dimensional spaces spanning the space of tensors of the form

$$
|| \begin{array}{lll}
a & b & 0 \\
b & c & 0 \\
0 & 0 & d
\end{array}||
$$

Here and below $\mathrm{a}, \mathrm{b}$ etc. are arbitrary numbers. The remaining irreducible spaces are also onedimensional, consisting of two orthogonal spaces, spanning the space indicated by

$$
|| \begin{array}{ccc}
0 & 0 & a \\
0 & 0 & b \\
a & b & 0
\end{array}||
$$

these being possibilities to which (4.3) applies.

No. 3

Cases to which (4.1) applies involve three one-dimensional spaces, with span of the form

$$
|| \begin{array}{lll}
a & 0 & 0 \\
0 & b & 0 \\
0 & 0 & c
\end{array}||
$$

The remaining irreducible spaces are also one-dimensional, those of the form

$$
|| \begin{array}{lll}
0 & a & 0 \\
0 & 0 & 0 \\
0 & 0 & 0
\end{array}||,|| \begin{array}{lll}
0 & 0 & a \\
0 & 0 & 0 \\
0 & 0 & 0
\end{array}||
$$


and

$$
|| \begin{array}{lll}
0 & 0 & 0 \\
0 & 0 & a \\
0 & 0 & 0
\end{array}||
$$

with (4.3) applying to these.

\section{No. 4}

Cases to which (4.1) applies involve two one-dimensional spaces, with span of the form

$$
|| \begin{array}{lll}
a & 0 & 0 \\
0 & a & 0 \\
0 & 0 & b
\end{array}||
$$

The remaining irreducible spaces consist of two two-dimensional spaces. One is of the form

$$
|| \begin{array}{ccc}
a & b & 0 \\
b & -a & 0 \\
0 & 0 & 0
\end{array}||
$$

If in it one takes as a basis

$$
\sqrt{2} \mathbf{E}_{1}=|| \begin{array}{ccc}
0 & 1 & 0 \\
1 & 0 & 0 \\
0 & 0 & 0
\end{array}|| \quad \sqrt{2} \mathbf{E}_{2}=\left|\begin{array}{ccc}
1 & 0 & 0 \\
0 & -1 & 0 \\
0 & 0 & 0
\end{array}\right| \mid
$$

(4.5) applies, with $\psi=2 \pi / 3$. The other is of the form

$$
|| \begin{array}{lll}
0 & 0 & a \\
0 & 0 & b \\
a & b & 0
\end{array}||
$$

and, with the basis 


$$
\sqrt{2} \mathbf{E}_{1}=|| \begin{array}{ccc}
0 & 0 & 1 \\
0 & 0 & 0 \\
1 & 0 & 0
\end{array}|| \quad \sqrt{2} \mathbf{E}_{2}=|| \begin{array}{ccc}
0 & 0 & 0 \\
0 & 0 & 1 \\
0 & 1 & 0
\end{array}||
$$

(4.5) applies, also with $\psi=2 \pi / 3$.

No. 5

Cases to which (4.1) applies involve two one-dimensional spaces, with span of the form

$$
|| \begin{array}{lll}
a & 0 & 0 \\
0 & a & 0 \\
0 & 0 & b
\end{array}||
$$

There are also two to which (4.3) applies, one of the form

$$
|| \begin{array}{lll}
0 & \mathrm{a} & 0 \\
\mathrm{a} & 0 & 0 \\
0 & 0 & 0
\end{array}||
$$

the other of the form

$$
|| \begin{array}{ccc}
\mathrm{a} & 0 & 0 \\
0 & -\mathrm{a} & 0 \\
0 & 0 & 0
\end{array}||
$$

Finally, there is one with dimension two, of the form

$$
|| \begin{array}{lll}
0 & 0 & a \\
0 & 0 & b \\
a & b & 0
\end{array}||
$$

With the basis 


$$
\sqrt{2} \mathrm{E}_{1}=|| \begin{array}{ccc}
0 & 0 & 1 \\
0 & 0 & 0 \\
1 & 0 & 0
\end{array}|| \quad, \quad \sqrt{2} \mathbf{E}_{2}=|| \begin{array}{ccc}
0 & 0 & 0 \\
0 & 0 & 1 \\
0 & 1 & 0
\end{array}||
$$

(4.5) applies, with $\psi=\pi / 2$.

No. 6

Cases to which (4.1) applies involve two one-dimensional spaces with span of the form

$$
|| \begin{array}{lll}
\mathrm{a} & 0 & 0 \\
0 & \mathrm{a} & 0 \\
0 & 0 & \mathrm{~b}
\end{array}||
$$

The other irreducible spaces are two-dimensional. One is of the form

$$
|| \begin{array}{ccc}
a & b & 0 \\
b & -a & 0 \\
0 & 0 & 0
\end{array}||
$$

with the basis

$$
\sqrt{2} \mathrm{E}_{1}=|| \begin{array}{lll}
0 & 1 & 0 \\
1 & 0 & 0 \\
0 & 0 & 0
\end{array}||, \quad \sqrt{2} \mathrm{E}_{2}=|| \begin{array}{ccc}
1 & 0 & 0 \\
0 & -1 & 0 \\
0 & 0 & 0
\end{array}||,
$$

(4.5) applies, with $\psi=2 \pi / 3$. The other is of the form

$$
|| \begin{array}{lll}
0 & 0 & a \\
0 & 0 & b \\
a & b & 0
\end{array}||
$$

with the basis 


$$
\sqrt{2} \mathbf{E}_{1}=|| \begin{array}{ccc}
0 & 0 & 1 \\
0 & 0 & 0 \\
1 & 0 & 0
\end{array}|| \quad \sqrt{2} \mathbf{E}_{2}=|| \begin{array}{lll}
0 & 0 & 0 \\
0 & 0 & 1 \\
0 & 1 & 0
\end{array}||
$$

(4.5) applies, this time with $\psi=\pi / 3$.

No. 7

Here, there is just one space to which (4.1) applies, of the form

$$
|| \begin{array}{lll}
\mathrm{a} & 0 & 0 \\
0 & \mathrm{a} & 0 \\
0 & 0 & \mathrm{a}
\end{array}||
$$

There is one of the two-dimensional kind, of the form

$$
|| \begin{array}{lll}
a & 0 & 0 \\
0 & b & 0 \\
0 & 0 & c
\end{array}|| ; a+b+c=0
$$

with

$$
\sqrt{2} \mathbf{E}_{1}=|| \begin{array}{ccc}
1 & 0 & 0 \\
0 & -1 & 0 \\
0 & 0 & 0
\end{array}|| \quad, \quad \sqrt{2} \mathbf{E}_{2}=|| \begin{array}{ccc}
1 & 0 & 0 \\
0 & 1 & 0 \\
0 & 0 & -2
\end{array}||
$$

(4.5) applies, with $\psi=2 \pi / 3$.

Finally, there is a three-dimensional space of the form

$$
|| \begin{array}{lll}
0 & a & b \\
a & 0 & c \\
b & c & 0
\end{array}||
$$

With the basis 


$$
\sqrt{2} \mathbf{E}_{1}=|| \begin{array}{ccc}
0 & 1 & 0 \\
1 & 0 & 0 \\
0 & 0 & 0
\end{array}|| \quad, \quad \sqrt{2} \mathbf{E}_{2}=|| \begin{array}{ccc}
0 & 0 & 0 \\
0 & 0 & 1 \\
0 & 1 & 0
\end{array}||
$$

and

$$
\sqrt{2} \mathbf{E}_{3}=|| \begin{array}{lll}
0 & 0 & 1 \\
0 & 0 & 0 \\
1 & 0 & 0
\end{array}||
$$

(4.7) applies.

So, this describes all of the likely choices of $\operatorname{ker} \boldsymbol{A}$, and links each one to one of six groups $\Re\left(\mathrm{P}_{0}\right)$. The next step is to deal with the bifurcation theory for each of the six.

\section{Cases with $\mathrm{N}=1$}

When $N=1$, we are concerned with the function $\Phi(y, \theta)$ discussed in Section 3 , y here being a scalar variable. As indicated in (3.8), we are given that it is a smooth function satisfying

$$
\frac{\partial \Phi}{\partial y}\left(0, \theta_{0}\right)=\frac{\partial^{2} \Phi}{\partial y^{2}}\left(0, \theta_{0}\right)=0
$$

with (5.1) 2 counting as one equation.

Suppose first that (4.1) applies, so that $\Phi$ is not subject to any invariance conditions. Then, what is likely is that

$$
\frac{\partial^{2} \Phi}{\partial y \partial \theta}\left(0, \theta_{0}\right) \neq 0
$$

for the vanishing of this would give a second equation. So we have here another use of our criterion. We can then use the implicit function theorem to solve the equilibrium equation 


$$
\frac{\partial \Phi}{\partial y}=0
$$

locally, for $\theta$, to get

$$
\theta=\theta_{0}+f(y), \quad f(0)=0, \quad f^{\prime}(0)=0
$$

the latter condition being obtained by calculating $f^{\prime}(0)$, using $(5.1)_{2}$. By a similar calculation, one finds that, most likely,

$$
f^{\prime \prime}(0) \neq 0 \text {, }
$$

so, near $y=0, f$ is either positive or negative, its graph resembling a parabola. If, say, $f<0$, one gets two equilibrium branches for $\theta<\theta_{0}$, coming together at $\theta=\theta_{0}$, to form one smooth curve in the $y-\theta$ plane. Since $\mathfrak{R}\left(\mathrm{P}_{0}\right)$ is here trivial, the orbit of a point on one of these branches consists of just one point. From the discussion in Section 3, this means that the point group there is the same as it is at the reference $y=0, \theta=\theta_{0}$ and, from the information in Section 4 , this can be any of the seven. So, this gives a bifurcation involving no change of symmetry. Also, what is likely is that $\partial^{2} \Phi / \partial y^{2}$ will be positive on one of these branches, negative on the other.

When (4.3) applies, $\Phi(y, \theta)$ is restricted to be an even function of y. What is then likely is the typical pitchfork bifurcation often encountered in the literature on bifurcation theory. Briefly, here

$$
y=0 \Rightarrow \frac{\partial \Phi}{\partial y}=0
$$

giving one equilibrium branch, passing smoothly through $\theta=\theta_{0}$. Since $y=0$ is a fixed point for this group, the point group on it is the same as that at the reference and, from the discussion in Section 4 , this could be No. 2,3 , or 5 . Besides the branch $y=0$, what is likely are branches defined either for $\theta \geq \theta_{0}$ or for $\theta \leq \theta_{0}$, describable by an equation of the form

$$
y^{2}=f(\theta), \quad f\left(\theta_{0}\right)=0, \quad f^{\prime}\left(\theta_{0}\right) \neq 0,
$$


where $f(\theta)$ is a smooth function. If, say, $f^{\prime}\left(\theta_{0}\right)>0$ and $\theta>\theta_{0}$, one will have an equilibrium point on one of these branches, with an orbit consisting of two points. The order of the point group at one of these will then be half of what it is at $y=0$. According to the Landau [7] exclusion rules, these are the bifurcations associated with likely second-order phase transitions for our Bravais lattices. Here, we do not preclude the possibility that all of these branches represent unstable equilibria, and they could not describe such transitions when they are. One can deduce something about the differences in sign of $\partial^{2} \Phi / \partial y^{2}$ on the different branches, but I won't elaborate this, which will be familiar to anyone who knows a bit about such bifurcations.

Essentially, these are textbook examples, encountered in various kinds of applications. I have elaborated them a bit, partly to illustrate how our criterion for being likely is used, to get more definite predictions. Also, we will see that some of the more complicated possibilities can be reduced to one of these cases. Further, if one looks at the analysis sketched, one sees that the smoothness assumptions on $\hat{\phi}$ are much stronger than is here necessary; to reduce smoothness assumptions to a minimum, one is likely to need to find alternatives to these and other arguments to be used.

Another point seems worth mentioning. In the case just discussed, a symmetry argument gave us the existence of one smooth branch $(y=0)$ passing through the bifurcation point. As it does, what is likely is that, on it, $\partial^{2} \Phi / \partial y^{2}$ will change sign; if it didn't, the partial derivative of this with respect to $\theta$ would vanish at bifurcation, giving a second equation. By an old result, due to Poincare $[8, \S 2]$, this implies the existence of equilibria with $y \neq 0$, in the neighborhood of the bifurcation point, and he discussed a generalization of this result to n-dimensional problems. This, combined with formal analysis, can be used to make rigorous arguments leading to (5.7), with fairly mild continuity assumptions. Very similar ideas underlie bifurcation analyses of cases yet to be considered. 
6. The case $\mathrm{N}=2, \psi=\pi / 2$

When $\mathrm{N}=2$, and $\Phi\left(\mathrm{y}_{1}, \mathrm{y}_{2}, \theta\right)$ is invariant under any of the groups $\mathrm{G}(\psi)$ described in (4.5) and (4.6), one has

$$
\lambda \frac{\partial \Phi}{\partial \mathbf{y}}(\mathbf{y}, \theta)=\frac{\partial \Phi}{\partial \mathbf{y}}(\lambda \mathbf{y}, \theta), \quad \lambda \in \mathrm{G}(\psi)
$$

with $\mathbf{y}=\left(\mathrm{y}_{1}, \mathrm{y}_{2}\right)$. These groups have in common the property that the only vector left invariant by the group is the null vector. From (6.1), we then infer that

$$
\mathbf{y}=0 \Rightarrow \frac{\partial \Phi}{\partial \mathbf{y}}=0,
$$

so there is at least this equilibrium branch, passing smoothly through the bifurcation point.

When $\psi=\pi / 2$, it is easy to verify that

$$
\Phi\left(\mathrm{y}_{1}, \mathrm{y}_{2}\right)=\Phi\left(-\mathrm{y}_{1}, \mathrm{y}_{2}\right)=\Phi\left(\mathrm{y}_{1},-\mathrm{y}_{2}\right)=\Phi\left(\mathrm{y}_{2}, \mathrm{y}_{1}\right)
$$

from which we have, in particular,

$$
\mathrm{y}_{1}=0 \Rightarrow \frac{\partial \Phi}{\partial \mathrm{y}_{1}}=0
$$

and

$$
\mathrm{y}_{2}=0 \Rightarrow \frac{\partial \Phi}{\partial y_{2}}=0
$$

and (6.5) can be obtained by transforming (6.4) by an element of $\mathrm{G}(\pi / 2)$. With (6.4), and $\mathrm{y}_{1}=0$, the equilibrium equations reduce to

$$
\frac{\partial \Phi}{\partial y_{2}}\left(0, y_{2}, \theta\right)=0
$$


$\Phi$ then being an even function of $\mathrm{y}_{2}$. Essentially, this is the second case discussed for $\mathrm{N}=1$. Thus we get the analog of (5.7), branches described by

$$
\mathrm{y}_{1}=0, \quad \mathrm{y}_{2}^{2}=\mathrm{f}(\theta), \quad \mathrm{f}\left(\theta_{0}\right)=0, \quad \mathrm{f}^{\prime}\left(\theta_{0}\right) \neq 0
$$

Transforming this gives the solution of (6.5)

$$
y_{2}=0, \quad y_{1}^{2}=f(\theta)
$$

Clearly, where $f$ is positive, the orbit of one equilibrium point like $(0, \sqrt{f(\theta)})$, consists of four distinct points. From Section 4 , the point group at $\mathbf{y}=0, \theta=\theta_{0}$ can only be No. 4 , configurations of the tetragonal kind, so (6.2) is a branch with this symmetry. From the Appendix, the order of the point group is eight. That on the symmetry-related branches described by (6.7) and (6.8) is then $8 / 4=2$, making this the point group No. 2 , the monoclinic configurations.

So, we have at least these branches. To explore the possibility of additional branching, we use (6.3), along with a little exercise in invariant theory, to infer that

$$
\Phi=F(I, J, \theta)
$$

where $\mathrm{F}$ is a smooth function of the variables

$$
\mathrm{I}=|\mathbf{y}|^{2}=\mathrm{y}_{1}^{2}+\mathrm{y}_{2}^{2}, \quad \mathrm{~J}=\mathrm{y}_{1}^{2} \mathrm{y}_{2}^{2}
$$

While our smoothness assumptions make it irrelevant, it is worth noting that, were $\Phi$ only a few times differentiable, $\mathrm{F}$ would be a bit less smooth, at some places, as follows from the results of Ball [9]. For any equilibrium points satisfying the condition

$$
\frac{\partial(\mathrm{I}, \mathrm{J})}{\partial\left(\mathrm{y}_{1}, \mathrm{y}_{2}\right)}=4 \mathrm{y}_{1} \mathrm{y}_{2}\left(\mathrm{y}_{1}^{2}-\mathrm{y}_{2}^{2}\right) \neq 0
$$

the equilibrium equations become

$$
\frac{\partial \mathrm{F}}{\partial \mathrm{I}}=\frac{\partial \mathrm{F}}{\partial \mathrm{J}}=0 \text {. }
$$


Suppose that there were a sequence of such equilibrium points, having $\mathbf{y}=0, \theta=\theta_{0}$ as a limit point. Then, by continuity, we would have

$$
\frac{\partial \mathrm{F}}{\partial \mathrm{I}}\left(0,0, \theta_{0}\right)=\frac{\partial \mathrm{F}}{\partial \mathrm{J}}\left(0,0, \theta_{0}\right)=0
$$

that is, at $\mathbf{y}=0, \theta=\theta_{0}$. To see whether this is likely, we can use the first few terms in an expansion of $\Phi$ about $\mathbf{y}=0$, viz.

$$
\Phi=\alpha+\beta I+\gamma \mathrm{J}+\delta \mathrm{I}^{2}+o\left(|\mathbf{y}|^{4}\right)
$$

where $\alpha, \beta$ etc. are functions of $\theta$. From our assumptions one equation must be satisfied, viz.

$$
\beta\left(\theta_{0}\right)=0
$$

so it is unlikely that $\gamma\left(\theta_{0}\right)=0$, i.e. that (6.13) is satisfied. So, we expect not to have equilibria of this kind. From (6.11), and our coverage of cases where $y_{1} y_{2}=0$, this leave the possibility of equilibria such that $\mathrm{y}_{1}^{2}=\mathrm{y}_{2}^{2} \neq 0$ and, by similar arguments, one can show that these are unlikely to occur.

7. The case $N=2, \psi=2 \pi / 3$

With $\Phi\left(\mathrm{y}_{1}, \mathrm{y}_{2}, \theta\right)$ invariant under the group $\mathrm{G}(2 \pi / 3)$ described by (4.5) and (4.6), we already have from (6.2), the branch

$$
\mathbf{y}=0
$$

and the easy observation that

$$
\mathrm{y}_{1}=0 \Rightarrow \frac{\partial \Phi}{\partial \mathrm{y}_{1}}=0
$$

This suggests looking at the possibility of satisfying

$$
\frac{\partial \Phi}{\partial y_{2}}\left(0, y_{2}, \theta\right)=0
$$


with $y_{2} \neq 0$. While this might seem to be the same as one of the two cases for which $\mathrm{N}=1$, it is not. For one thing, one can differentiate (6.1) with respect to $\theta$ to get, in particular,

$$
\frac{\partial^{2} \Phi}{\partial y_{2} \partial \theta}(0,0, \theta)=0
$$

a consequence of symmetry. This makes it clear that this is unlike the first case discussed in Section 2. Also, it is easy to check that $\Phi\left(0, y_{2}, \theta\right)$ is here not an even function of $y_{2}$, making it unlike the second case.

By using our now-familiar criterion, it is easy to check that, most likely,

$$
\frac{\partial^{3} \Phi}{\partial y_{2}^{2} \partial \theta}\left(0,0, \theta_{0}\right) \neq 0
$$

So, on the branch (7.1), $\partial^{2} \Phi / \partial y_{2}^{2}$ will change sign, as $\theta$ passes through the value $\theta_{0}$. By applying the result of Poincaré, mentioned at the end of Section 5 , to $\Phi\left(0, y_{2}, \theta\right)$, one infers the existence of solutions of (7.3) with $y_{2} \neq 0$, in the neighborhood of $y_{2}=0, \theta=\theta_{0}$. I won't expend the work required to describe my elementary analysis of these, but it produces one branch described by

$$
y_{1}=0, \quad y_{2}=f(\theta), \quad f\left(\theta_{0}\right)=0, \quad f^{\prime}\left(\theta_{0}\right) \neq 0
$$

Transforming this by elements of the group gives two more, of the form

$$
2 \mathrm{y}_{1}= \pm \sqrt{3} \mathrm{f}(\theta), \quad 2 \mathrm{y}_{2}=-\mathrm{f}(\theta)
$$

or three symmetry-related branches in all.

To explore whether occurrence of additional branches is likely, we note that the invariance of $\Phi$ under $\mathrm{G}(2 \pi / 3)$ implies that it is an even function of $y_{1}$, with $y_{2}$ fixed. Thus, we can regard it as a smooth function of $y_{1}^{2}$ and $y_{2}$ or, equivalently, of the variables

$$
X=y_{1}^{2}-3 y_{2}^{2}, Y=y_{2} \text {, }
$$


so

$$
\Phi\left(\mathrm{y}_{1}, \mathrm{y}_{2}, \theta\right)=\mathrm{F}(\mathrm{X}, \mathrm{Y}, \theta)
$$

a function which is still subject to some invariance requirements. What we have above are equilibria on the lines $\mathrm{y}_{1}=0$, and $\mathrm{y}_{1}= \pm \sqrt{3} \mathrm{y}_{2}$, so let us look for equilibria occurring elsewhere with, say, $\theta>\theta_{0}$. If $f(\theta)$ is then positive, we look at the domain described by

$$
\mathrm{y}_{2}<0, \quad \sqrt{3} \mathrm{y}_{2}<\mathrm{y}_{1}<0
$$

If we have any equilibrium point not on one of the three lines, some point on its orbit will be in this domain. In the $\mathrm{X}-\mathrm{Y}$ plane, this maps to

$$
\mathrm{Y}<0,-3 \mathrm{Y}^{2}<\mathrm{X}<0
$$

Here, we are interested in possible solutions of

$$
\frac{\partial \mathrm{F}}{\partial \mathrm{X}}=\frac{\partial \mathrm{F}}{\partial \mathrm{Y}}=0
$$

and know that they are satisfied at $(0,-f(\theta) / 2)$. Suppose that, at $\theta=\theta_{1}$, they are also satisfied at $\left(X_{1}, Y_{1}\right) \neq\left(0,-f\left(\theta_{1}\right) / 2\right)$ and consider the function

$$
\mathrm{g}(\tau)=\frac{\partial \mathrm{F}}{\partial \mathrm{X}}\left[\tau \mathrm{X}_{1}, \tau \mathrm{Y}_{1}+(\tau-1) \mathrm{f}\left(\theta_{1}\right) / 2, \theta_{1}\right]
$$

it vanishes at $\tau=0$ and at $\tau=1$ so, for some $\tau \in(0,1), \mathrm{g}^{\prime}(\tau)=0$. This gives us a point at which

$$
\frac{\partial^{2} F}{\partial X^{2}} X_{1}+\frac{\partial^{2} F}{\partial X \partial Y}\left(Y_{1}+f\left(\theta_{1}\right) / 2\right)=0
$$

In a similar way, by using $\partial \mathrm{F} / \partial \mathrm{Y}$, we can get a point, generally different, at which

$$
\frac{\partial^{2} F}{\partial X \partial Y} \cdot X_{1}+\frac{\partial^{2} F}{\partial Y^{2}}\left(Y_{1}+f\left(\theta_{1}\right) / 2\right)=0
$$

Here, we can replace $\left(X_{1}, Y_{1}+f\left(\theta_{1}\right) / 2\right)$ by the unit vector 


$$
\left(X_{1} / r,\left(Y_{1}+f\left(\theta_{1}\right) / 2\right) / r\right), r=\left|\left(X_{1}, Y_{1}+f\left(\theta_{1}\right) / 2\right)\right|
$$

Now, suppose that there were a sequence of such equilibria, having as a limit point $(0,0)$ as $\theta \rightarrow \theta_{0}$. This will give a sequence of points on the unit circle which must have some limit point. Also, the points where (7.14) and (7.15) hold will tend to $(0,0)$ as $\theta \rightarrow \theta_{0}$. Putting this together, one gets the condition

$$
\frac{\partial^{2} F}{\partial X^{2}} \frac{\partial^{2} F}{\partial Y^{2}}-\left(\frac{\partial^{2} F}{\partial X \partial Y}\right)^{2}=0 \quad \text { at } X=Y=0, \theta=\theta_{0}
$$

Now, with a little exercise in invariant theory, one gets the approximation

$$
\Phi=\alpha+\beta|\mathbf{y}|^{2}+\gamma y_{2}\left(3 y_{1}^{2}-y_{2}^{2}\right)+\delta|\mathbf{y}|^{4}+o\left(|y|^{4}\right)
$$

or

$$
\mathrm{F}(\mathrm{x}, \mathrm{y}, \theta) \cong \alpha+\beta\left(\mathrm{X}+4 \mathrm{Y}^{2}\right)+\gamma \mathrm{Y}\left(3 \mathrm{X}-4 \mathrm{Y}^{2}\right)+\delta\left(\mathrm{X}+4 \mathrm{Y}^{2}\right)^{2}
$$

where $\alpha, \beta$, etc. are functions of $\theta$. From our assumptions, $\beta\left(\theta_{0}\right)$ must vanish, one equation to be satisfied. For (7.17) to hold would require that $\gamma\left(\theta_{0}\right)$ also vanish, so this is unlikely. With slight changes in the argument, one can cover the possibility that $f(\theta)<0$ for $\theta>\theta_{0}$, and cover equilibria which might occur for $\theta<\theta_{0}$. In brief, what is likely is that we will have the branches described by (7.1), (7.6) and (7.7), and no others. Physically, these could be of the trigonalmonoclinic hexagonal-orthorhombic or cubic-tetragonal kind. The latter possibility is discussed in more detail in Section 10.

\section{The case $N=2, \psi=\pi / 3$}

With $\Phi\left(y_{1}, y_{2}, \theta\right)$ invariant under $G(\pi / 3)$, we again have the trivial branch

$$
\mathbf{y}=0 \text {, }
$$

for a start. Notice that this group includes rotations with angle $\pi$, and you see that $\Phi$ satisfies

$$
\Phi\left(\mathrm{y}_{1}, \mathrm{y}_{2}, \theta\right)=\Phi\left(-\mathrm{y}_{1}, \mathrm{y}_{2}, \theta\right)=\Phi\left(\mathrm{y}_{1},-\mathrm{y}_{2}, \theta\right)
$$


as well as some additional conditions. One oddity is that one has to go to approximations of rather high order, to get polynomials which are invariant under this group, and not the full orthogonal group; my calculations give

$$
\Phi=\alpha+\beta|\mathbf{y}|^{2}+\gamma|\mathbf{y}|^{4}+\delta|\mathbf{y}|^{6}+\epsilon\left(y_{1}^{2}-y_{2}^{2}\right)\left[4\left(y_{1}^{2}-y_{2}^{2}\right)-3|y|^{4}\right]+o\left(|y|^{6}\right)
$$

where $\alpha, \beta$, etc. are functions of $\theta$. Still, $\Phi\left(0, y_{2}, \theta\right)$ is a rather general even function of $y_{2}$ and, as was the case for $G(\pi / 2)$, we get branches of the typical pitchfork kind

$$
y_{1}=0, \quad y_{2}^{2}=f(\theta), \quad f\left(\theta_{0}\right)=0, \quad f^{\prime}\left(\theta_{0}\right) \neq 0 \text {. }
$$

Transforming this by the elements of the group has the effect of rotating this by angles, $n \pi / 3, n=1$, $2,3,4,5$. Since (8.3) describes two equilibria when $f>0$, the orbit consists of twelve distinct points. Checking the information you have been given, in Section 4 and the Appendix, you find that, so far, what we have is a hexagonal-triclinic bifurcation. By essentially the same reasoning, one can get a similar but different array of branches, in the orbit of one of the form

$$
y_{2}=0, \quad y_{1}^{2}=g(\theta), \quad g\left(\theta_{0}\right)=0, \quad g^{\prime}\left(\theta_{0}\right) \neq 0 \text {. }
$$

a fact which seems to me rather curious.

Here, it is convenient to regard $\Phi$ as a somewhat special function of the variables

$$
\mathrm{K}=|\mathbf{y}|^{2}, \quad \mathrm{~L}=\mathrm{y}_{1}^{2}-\mathrm{y}_{2}^{2} \Rightarrow|\mathrm{L}| \leq \mathrm{K}, \quad \mathrm{K}>0
$$

and $\theta$,

$$
\Phi=F(K, L, \theta)
$$

(8.2) serving to justify this. In terms of these, some of the equilibria described above lie on the lines in the K-L plane given by

$$
\mathrm{L}= \pm \mathrm{K} \text {. }
$$


If there are any other kinds, they will be in the sector

$$
\mathrm{K}>0, \quad|\mathrm{~L}|<\mathrm{K} .
$$

Suppose that there is one there, at $\left(\mathrm{K}_{0}, \mathrm{~L}_{0}\right)$, at $\theta=\theta_{1}$. By a routine calculation to determine others on the same orbit, one gets them at

$$
\left(\mathrm{K}_{0},-\mathrm{L}_{0} / 2 \pm \mathrm{M}_{0}\right), 4 \mathrm{M}_{0}^{2}=3\left(\mathrm{~K}_{0}^{2}-\mathrm{L}_{0}^{2}\right) \text {. }
$$

Now, when the three points indicated are distinct, repeated use of the mean value theorem yields the condition

$$
\frac{\partial^{3} \mathrm{~F}}{\partial \mathrm{L}^{3}}\left(\mathrm{~K}_{1}, \mathrm{~L}_{1}, \theta_{1}\right)=0
$$

$\left(\mathrm{K}_{1}, \mathrm{~L}_{1}\right)$ being some point in the sector. So, if there is a sequence of these approaching $(0,0)$ as $\theta_{1} \rightarrow \theta_{0}$, we must have

$$
\frac{\partial^{3} \mathrm{~F}}{\partial \mathrm{L}^{3}}\left(0,0, \theta_{0}\right)=0 .
$$

With (8.3), this gives $\epsilon\left(\theta_{0}\right)=0$ and, by now familiar reasoning, this is unlikely.

Within the sector, $\mathrm{M}_{0} \neq 0$, so, for two of the points to coincide, we must have

$$
\mathrm{L}_{0}=-\mathrm{L}_{0} / 2 \pm \mathrm{M}_{0} \text {, }
$$

in which case there are two distinct points. By a routine calculation, these correspond to points on the lines

$$
y_{1}= \pm \sqrt{3} y_{2}, \quad y_{2}= \pm \sqrt{3} y_{1}
$$

lines obtained by taking orbits of the lines $y_{1}=0$ and $y_{2}=0$. So, there are such equilibria, but they have been accounted for. 
Here, particularly, I find the analyses unpleasant, in that they presume much differentiability of $\hat{\phi}$. Perhaps some nicer treatment is in the literature, but I haven't found one.

\section{The case $\mathrm{N}=3$}

Here, we are dealing with functions $\Phi\left(\mathrm{y}_{1}, \mathrm{y}_{2}, \mathrm{y}_{3}, \theta\right)$, which are invariant under the large group $\mathrm{G}(3)$ with generators described in (4.8). Again, the only vector left invariant by this group is the null vector, so we have the branch implied by

$$
\mathbf{y}=0 \Rightarrow \frac{\partial \Phi}{\partial \mathbf{y}}=0
$$

passing smoothly through $\mathbf{y}=0, \theta=\theta_{0}$, configurations of the cubic kind. The invariance implies that, when any one of the coordinates vanishes, $\Phi$ reduces to an even function of the other two, whence follows that

$$
\mathrm{y}_{2}=\mathrm{y}_{3}=0 \Rightarrow \frac{\partial \Phi}{\partial \mathrm{y}_{2}}=\frac{\partial \Phi}{\partial \mathrm{y}_{3}}=0, \text { etc. }
$$

with $\Phi\left(y_{1}, 0,0, \theta\right)$ an even function of $y_{1}$. After checking the invariance requirements on it, one finds that these don't exclude the occurrence of the typical pitchfork bifurcations, so we will have branches described by

$$
y_{2}=y_{3}=0, \quad y_{1}^{2}=f(\theta), \quad f\left(\theta_{0}\right)=0, \quad f^{\prime}\left(\theta_{0}\right) \neq 0
$$

The orbit of this gives two more "copies," obtained by replacing y 1 by $y_{2}$ and by $y_{3}$. So, where $f(\theta)>0$, the orbit of one such equilibrium point contains six distinct points. The order of the cubic group is 24 , so the order of the point group on any of these branches is $24 / 6=4$, identifying this as the orthorhombic group. Here, at least when $\Phi$ is a polynomial, it is known that it can be shown to be representable in the form

$$
\Phi=\mathrm{QF}_{1}+\mathrm{K} \Phi_{2}, \quad \mathrm{~K}=\mathrm{y}_{1} \mathrm{y}_{2} \mathrm{y}_{3}
$$

with $\Phi_{1}$ and $\Phi_{2}$ symmetric functions of $y_{1}^{2}, y_{2}^{2}$ and $y_{3}^{2}$, expressible as functions of 


$$
\begin{aligned}
I & =y_{1}^{2}+y_{2}^{2}+y_{3}^{2}, \\
J & =y_{1}^{4}+y_{2}^{4}+y_{3}^{4}, \\
\text { and } K^{2} & =\left(y_{1} y_{2} y_{3}\right)^{2}
\end{aligned}
$$

Or, more simply, we have

$$
\Phi=\mathrm{F}(\mathrm{I}, \mathrm{J}, \mathrm{K}, \theta)
$$

where $\mathrm{F}$ is a polynomial in I, J, $\mathrm{K}^{*}$. In fact, one can adapt the analysis by Ball [9] to justify our assuming that (9.6) holds, with $\mathrm{F}$ a smooth function. At points such that

$$
\frac{\partial(I, J, K)}{\partial\left(y_{1}, y_{2}, y_{3}\right)}=8\left(y_{1}^{2}-y_{2}^{2}\right)\left(y_{2}^{2}-y_{3}^{2}\right)\left(y_{3}^{2}-y_{1}^{2}\right) \neq 0
$$

the equilibrium equations are

$$
\frac{\partial \mathrm{F}}{\partial \mathrm{I}}=\frac{\partial \mathrm{F}}{\partial \mathrm{J}}=\frac{\partial \mathrm{F}}{\partial \mathrm{K}}=0
$$

and it is easy enough to show that it is unlikely that these hold at $\mathbf{y}=0, \theta=\theta_{0}$, so, by reasoning now familiar, we can dismiss such equilibria. From (9.7), this leaves unsettled the possibilities like

$$
\mathrm{y}_{2}= \pm \mathrm{y}_{3} \neq 0
$$

From above, it is easy to see that the restriction of $\Phi$ to the set

$$
\mathrm{y}_{2}=\mathrm{y}_{3},
$$

say, is an even function of this variable, so we can regard it as a function of its square, so

$$
\Phi\left(y_{1}, y_{2}, y_{2}, \theta\right)=G(X, Z, \theta)
$$

with

$$
\mathrm{X}=\mathrm{y}_{1}, \quad \mathrm{Z}=\mathrm{y}_{2}^{2} .
$$

* This can be inferred from results of Smith and Rivlin [10], for example. 
From, say, (9.5) and (9.6), it is clear that

$$
\Phi=\alpha+\beta I+\gamma \mathrm{K}+\delta \mathrm{I}^{2}+\epsilon \mathrm{J}+\mathrm{o}\left(|\mathbf{y}|^{4}\right),
$$

where $\alpha, \beta$, etc. are functions of $\theta$,

$$
\beta\left(\theta_{0}\right)=0
$$

being the one equation we allow. From this we have the approximation

$$
G \cong \alpha+\beta\left(X^{2}+2 Z\right)+\gamma X K+\delta\left(X^{2}+2 Z\right)^{2}+\epsilon\left(X^{4}+2 Z^{2}\right),
$$

Also, for equilibria with $\mathrm{Z}>0$, conditions of equilibria imply that

$$
\frac{\partial G}{\partial X}=\frac{\partial G}{\partial Z}=0 \text {. }
$$

With (9.15), it is easy to verify that these are satisfied at $X=0, Z=0, \theta=\theta_{0}$. Also, here,

$$
\operatorname{det}|| \begin{array}{cc}
\frac{\partial^{2} G}{\partial X^{2}} & \frac{\partial^{2} G}{\partial X \partial Z} \\
\frac{\partial^{2} G}{\partial X \partial Z} & \frac{\partial^{2} G}{\partial Z^{2}}
\end{array}||=-\gamma^{2}\left(\theta_{0}\right)
$$

which is not likely to vanish. So, the implicit function theorem applies, giving us the existence and uniqueness of solutions of (9.16), locally. In first approximation, we get the solution as

$$
\left.\begin{array}{l}
X \cong-2 \beta^{\prime}\left(\theta_{0}\right)\left(\theta-\theta_{0}\right) / \gamma\left(\theta_{0}\right), \\
Z \cong 4\left[\beta^{\prime}\left(\theta_{0}\right)\right]^{2}\left(\theta-\theta_{0}\right)^{2} / \gamma\left(\theta_{0}\right)^{2},
\end{array}\right\}
$$

which is consistent with having $Z>0$, as it should be. Really, there are three equilibrium equations to be satisfied, in general. However, it is easy to check that

$$
\mathrm{y}_{1}=\mathrm{y}_{1} \Rightarrow \frac{\partial \Phi}{\partial \mathrm{y}_{1}}=\frac{\partial \Phi}{\partial \mathrm{y}_{2}}
$$


one can use (9.6) to confirm this, for example, as well as checking that it is sufficient to satisfy (9.16). Now (9.18) suggests that, for these solutions, $Z=X^{2}$, and it isn't hard to verify this, using the uniqueness of solutions. So, this gives us equilibria of the form

$$
y_{1}=f(\theta), \quad y_{2}=y_{3}, \quad y_{2}^{2}=f^{2}(\theta)
$$

(9.18) giving a first approximation of $f(\theta)$. Of course, it is unlikely that $\beta^{\prime}\left(\theta_{0}\right)=0$. Naturally, G(3) generates an orbit of "copies" of (9.20), which covers the prossibilities of having any two of the three quantities $y_{1}^{2}, y_{2}^{2}, y_{3}^{2}$ be equal and nonzero, and we have also covered the cases where some or all vanish. Counting up the number of distinct points on an orbit of this kind, I find four. So the order of the point group for these is $24 / 4=6$, the trigonal type. So, we have quite a variety of branches, in this case. I do not know of observations of a softening modulus which would suggest a bifurcation of this kind. However, there aren't very many observations of this general kind, and there are hardly any for Martensitic phases, which here could be either of the orthorhombic or the trigonal kind, for suitable forms of the function $\hat{\phi}$.

\section{Example}

For shape-memory alloys, one of the more commonly observed possibilities has as Austenite a crystal of cubic form. Since this group is maximal, any limit point is also cubic. Upon cooling it, the observations indicate softening described by

$$
\mathrm{C}_{11}-\mathrm{C}_{12} \rightarrow 0
$$

in notation commonly used by experimentalists and by Love [11], for example. Here the C's are not to be confused with components of the tensor $\mathrm{C}$. Referring to the comments about the cubic (No. 7) in Section 4, the eigenvalue which seems to vanish is that associated with the twodimensional eigenspace, giving us the case $N=2, \mathfrak{R}\left(P_{0}\right)=G(2 \pi / 3)$ considered in Section 7: the observations give that the other two eigenvalues remain positive. From the discussion in Section 
7, the cubic branch passes smoothly through $\theta=\theta_{0}$, with the indicated eigenvalue becoming negative, for $\theta<\theta_{0}$, or, said differently,

$$
\mathrm{C}_{11}-\mathrm{C}_{12}<0 \text { for } \theta<\theta_{0} \text {. }
$$

Also, we have the three symmetry-related branches noted in (7.6) and (7.7), involving the tetragonal symmetry. One of these will match the description in the Appendix, with the same basis $\mathbf{e}_{1}, \mathbf{e}_{2}, \mathbf{e}_{3}$ used for the tetragonal and the cubic configurations. From Section $4, a$ generally has five distinct values for tetragonal crystals, but at the bifurcation point one has the cubic symmetry so, as a consequence of invariance, some of the five must become equal to match the three for the cubic. This provides an example of an exceptional case, not regarded as unlikely, in which normally distinct eigenvalues can become equal and also vanish. Looking at what is involved, we find that, for the tetragonal, the eigenvalues associated with the two-dimensional eigenspace and the one-dimensional space of the form

$$
|| \begin{array}{lll}
0 & a & 0 \\
a & 0 & 0 \\
0 & 0 & 0
\end{array}||
$$

become equal to a positive eigenvalue for the cubic, so both of these are positive, locally. Of the two eigenvalues with span of the form

$$
|| \begin{array}{lll}
a & 0 & 0 \\
0 & a & 0 \\
0 & 0 & b
\end{array}||
$$

one approaches that associated with the one-dimensional eigenspace for the cubic and is therefore positive. The other merges with that for which the eigenspace is of the form

$$
|| \begin{array}{ccc}
\mathrm{a} & 0 & 0 \\
0 & -\mathrm{a} & 0 \\
0 & 0 & 0
\end{array}|| \text {, }
$$


these vanishing at the bifurcation point. With some additional analysis, which I won't elaborate, one can show that, nearby, one of the two is positive, one negative, with both of these changing sign as $\theta$ passes through $\theta_{0}$, indicating that these branches are unstable, having one negative eigenvalue, associated with a one-dimensional eigenspace. Actually, that associated with (10.5) is positive for $\theta>\theta_{0}$. There are simple possibilities for such branches regaining stability. For example, for $\theta>\theta_{0}$, the negative eigenvalue is one of those with the span (10.3), and it could become zero at some such temperature. From Section 4, this would give a bifurcation for which $\mathrm{N}=1$, the first kind discussed in Section 5. That is, one would have a tetragonal branch with positive eigenvalues, for temperatures below the temperature at which this bifurcation occurs. Exchange of stability between this and the cubic would then produce a rather typical subcritical bifurcation, a cubic-tetragonal phase transition of first order, and transitions of this general kind occur in shape-memory alloys. If this picture applies, one has a prediction that a certain eigenvalue associated with the tetragonal should become relatively small, near the indicated bifurcation. It is at least within the realm of possibility that experiments could confirm or deny this: I have not seen such data yet, but expect that experimentalists will produce some eventually. It isn't hard to see that more complicated combinations of bifurcations can be associated with cubic-tetragonal transitions and predict softening of the same tetragonal modulus. Simply, it is not very clear what can be done, experimentally, to discern between such possibilities, but this is a question which seems to deserve thoughtful consideration.

With the information provided, an interested reader can similarly treat the possibility that the negative eigenvalue which again vanishes is that associated with (10.5), which gives a theoretically possible picture of a cubic-orthorhombic transition of first order, associated with softening of the same cubic modulus. Again, there are observations of cubic-orthorhombic transitions associated with this kind of softening in shape-memory alloys, but there is a quirk. What the suggested analysis gives could fit some kinds of cubic-orthorhombic transitions, but not one of the body-centered-cubic-face-centered-orthorhombic type, for example; this can be inferred 
by calculating the form of $\mathbf{C}$ associated with the orthotropic phase. On the other hand, transitions of this kind are observed. If nothing else, this indicates that it can be feasible to prove wrong a simple guess about a global bifurcation pattern, with the rather limited experimental information now available. More accurately, I am inclined to believe that the general theory does apply to such transitions, it being the guess about the global bifurcation pattern which is at fault. After seeing what is wrong, one can make better educated guesses about the global pattern, finding some which could fit such transitions. One might find reason to reject some such, given experimental information concerning what is the soft modulus in the orthorhombic phase.

This discussion serves to illustrate some of the kinds of things that can be inferred about unstable branches, using kinds of experimental results which could now be obtained, by knowledgeable experimentalists.

I see this as a small piece of a larger problem. Among workers interested in phase transitions, it is a rather common practice to make some guess about the form of the relevant themodynamic potential, involving some adjustable parameters which can be determined, using data which either has been or obviously can be obtained. Roughly, the general problem is to find the qualities characterizing the more likely guesses, and I expect some of these to be of a qualitative nature. For the more mathematical studies, what is often important is not the specific form of our function $\hat{\phi}$, say, but of its qualitative features. Looking back at our analyses, we have used some polynomial approximations of fairly high degree, and such polynomials represent popular guesses as to the form of $\hat{\phi}$. From the work of Smith and Rivlin [10], one can determine those which are invariant under any one of the point groups. My experience is that you will have trouble finding relevant data to determine all of the adjustable constants involved. Even if you find enough data to determine all, you are likely to have a complicated function, making it hard to see just what general properties it has, that might enable you to apply some mathematical theorem. Thinking about this persuaded me that it was worthwhile to develop the kinds of pictures which I have presented here. 
Those who wonder why I have not considered crystals of more than general kinds might consult the paper by Zanzotto [12]; there are some good physical reasons for this.

\section{Appendix}

The (three-dimensional) crystallographic groups, being subgroups of the orthogonal group $\mathrm{O}(3)$, have elements $\mathbf{Q} \in \mathrm{O}(3)$, representable in the form

$$
Q= \pm R ，
$$

where

$$
\mathbf{R}=\mathbf{R}(\psi, \mathbf{e}) \in \mathrm{SO}(3)
$$

is a rotation with angle $\psi$, $\mathbf{e}$ being a unit vector, representing the axis of rotation. The point groups realizable in Bravais lattices all contain the central inversion $Q=-1$, so can be generated by this and some group consisting of rotations. What is referred to as the point group $\mathrm{P}_{0}$ in this paper is the latter group. Strictly speaking, this is not correct, but I don't think that this will cause confusion.

Involved in the description of each is an orthonormal basis $\mathbf{e}_{i}(i=1,2,3)$, vectors often referred to as crystallographic axes. Where you see the designation $\mathbf{e}=\mathbf{p}$, this means to use for $\mathbf{e}$ all of the four vectors

$$
\left(\mathbf{e}_{1} \pm \mathbf{e}_{2} \pm \mathbf{e}_{3}\right) / \sqrt{3} \text {. }
$$

Listed according to their order, the indicated groups are 


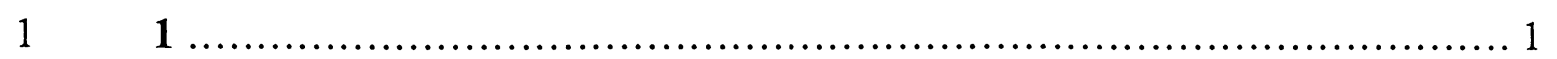

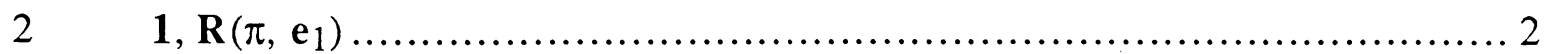

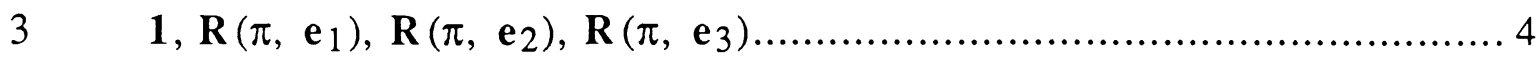

$4 \quad 1 ; \mathbf{R}\left(\pi, \mathbf{e}_{1}\right), \mathbf{R}\left(\pi, \frac{\mathbf{e}_{1} \pm \sqrt{3} \mathbf{e}_{2}}{2}\right)$,

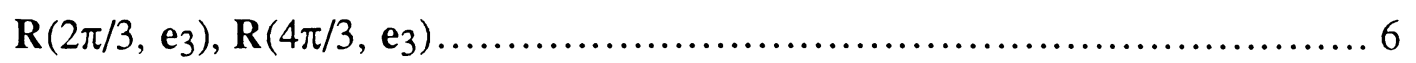

$5 \quad \mathbf{1}, \mathbf{R}\left(\pi, \mathrm{e}_{1}\right), \mathbf{R}\left(\pi, \mathrm{e}_{2}\right), \mathbf{R}\left(\pi, \mathrm{e}_{3}\right)$,

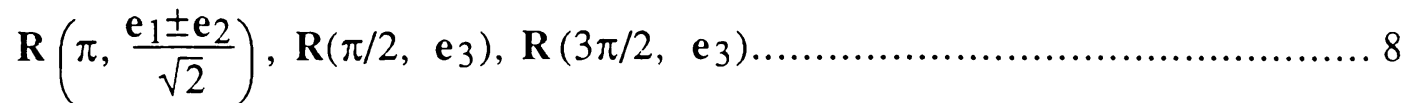

$6 \quad \mathbf{1}, \mathbf{R}\left(\pi, \mathrm{e}_{1}\right), \mathbf{R}\left(\pi, \mathrm{e}_{2}\right), \mathbf{R}\left(\pi, \mathrm{e}_{3}\right)$,

$\mathbf{R}\left(\pi, \frac{\mathbf{e}_{1} \pm \sqrt{3} \mathbf{e}_{2}}{2}\right), \mathbf{R}\left(\pi, \frac{\sqrt{3} \mathbf{e}_{1} \pm \mathbf{e}_{2}}{2}\right), \mathbf{R}\left(\pi / 3, \mathbf{e}_{3}\right)$,

$\mathbf{R}\left(2 \pi / 3, \mathbf{e}_{3}\right), \mathbf{R}\left(4 \pi / 3, \mathbf{e}_{3}\right), \mathbf{R}\left(5 \pi / 3, \mathbf{e}_{3}\right)$

$7 \quad \mathbf{1}, \mathbf{R}\left(\pi / 2, \mathbf{e}_{1}\right), \mathbf{R}\left(\pi / 2, \mathbf{e}_{2}\right), \mathbf{R}\left(\pi / 2, \mathbf{e}_{3}\right)$, $\mathbf{R}\left(\pi, \frac{\mathbf{e}_{1} \pm \mathrm{e}_{2}}{\sqrt{2}}\right), \mathbf{R}\left(\pi, \frac{\mathbf{e}_{2} \pm \mathrm{e}_{3}}{\sqrt{2}}\right), \mathbf{R}\left(\pi, \frac{\mathbf{e}_{1} \pm \mathrm{e}_{3}}{\sqrt{2}}\right)$,

$\mathbf{R}\left(\pi, \mathbf{e}_{1}\right), \mathbf{R}\left(\pi, \mathbf{e}_{2}\right), \mathbf{R}\left(\pi, \mathbf{e}_{3}\right)$,

$\mathbf{R}\left(3 \pi / 2, \mathbf{e}_{1}\right), \mathbf{R}\left(3 \pi / 2, \mathbf{e}_{2}\right), \mathbf{R}\left(3 \pi / 2, \mathbf{e}_{3}\right)$,

$\mathbf{R}(2 \pi / 3, \mathbf{p}), \mathbf{R}(4 \pi / 3, \mathbf{p})$

Crystals having symmetries described by one of these form one of the crystal systems or classes, as they are sometimes called. Different workers use different names for these, those used here being as follows:

1. Triclinic

2. Monoclinic

3. Orthorhombic

4. Trigonal

5. Tetragonal

6. Hexagonal

7. Cubic 
One can further refine these into different symmetry types. As is discussed by Ericksen [4], this is of some importance for understanding what kinds of bifurcations are possible, but, here, it is not necessary to make any explicit use of these refinements. One could specify the symmetry type at bifurcation, then infer something about the symmetry types on branches, for any of the cases here considered. By not doing so, I have left the theory somewhat incomplete. For those familiar with such matters, I don't think that it would be hard to fill in details of this kind.

\section{Acknowledgement}

This work was supported by AFOSR/XOP and NSF, under grant NSF/DMS 8718881.

\section{References}

1. Material Instabilities in Continuum Mechanics (ed. J.M. Ball), Oxford University Press (1988)

2. Shape Memory Effects in Alloys (ed. J. Perkins), Plenum Press, New York-London (1975)

3. Ericksen, J.L., Bifurcation and Martensitic transformations in Bravais lattices, to appear in J. Elasticity.

4. Ericksen, J.L., Weak Martensitic transformations in Bravais lattices, Arch. Ration. Mech. Anal. 107 (1989), pp. 23-36.

5. Truesdell, C., First Course in Rational Mechanics, vol. 1, Academic Press, New York-San Francisco-London (1977)

6. Man, C.-S., Material stability, the Gibbs conjecture and the first phase rule for substances, Arch. Ration. Mech. Anal. 91 (1985), pp. 1-53. 
7. Landau, L.D., On the theory of phase transitions, in Collected Papers of L.D. Landau (ed. D. TerHaar) Gordon and Breach, and Pergamon Press, New York-London-Paris (1965)

8. Poincaré, H., Sur l'Équilibre d'une mass fluide animée d' une mouvement de rotation, Acta Math. 7 (1885), pp. 259-380.

9. Ball, J.M., Differentiability properties of symmetric and isotropic functions, Duke Math. J. 51 (1984), pp. 699-728.

10. Smith, G.F. and Rivlin, R.S., The strain-energy function for anisotropic elastic materials, Trans. Am. Math. Soc. 88 (1958), pp. 175-193.

11. Love, A.E.H., A Treatise on the Mathematical Theory of Elasticity, 4th ed., Cambridge University Press (1927)

12. Zanzotto, G., Twinning in minerals and metals: remarks on the comparison of $a$ thermoelasticity theory with some available experimental results, to appear in Rend. dell' Accad. Lincei. 
Philip Korman, Dynamics of the Lotka-Volterra systems with diffusion

Harlan W. Stech, Generic Hopf bifurcation in a class of integro-differential equations

Stephane Laederich, Periodic solutions of non linear differential difference equations

Peter J. Olver, Canonical Forms and Integrability of BiHamiltonian Systems

S.A. van Gils, M.P. Krupa and W.F. Langford, Hopf bifurcation with nonsemisimple 1:1 Resonance

R.D. James and D. Kinderlehrer, Frustration in ferromagnetic materials

Carlos Rocha, Properties of the attractor of a scalar parabolic P.D.E.

Debra Lewis, Lagrangian block diagonalization

Richard C. Churchill and David L. Rod, On the determination of Ziglin monodromy groups

Xinfu Chen and Avner Friedman, A nonlocal diffusion equation arising in terminally attached polymer chains

Peter Gritzmann and Victor Klee, Inner and outer j- Radii of convex bodies in finitedimensional normed spaces

P. Szmolyan, Analysis of a singularly perturbed traveling wave problem

Stanley Reiter and Carl P. Simon, Decentralized dynamic processes for finding equilibrium

Fernando Reitich, Singular solutions of a transmission problem in plane linear elasticity for wedge-shaped regions

Russell A. Johnson, Cantor spectrum for the quasi-periodic Schrödinger equation

Wenxiong Liu, Singular solutions for a convection diffusion equation with absorption

Deborah Brandon and William J. Hrusa, Global existence of smooth shearing motions of a nonlinear viscoelastic fluid

James F. Reineck, The connection matrix in Morse-Smale flows II

Claude Baesens, John Guckenheimer, Seunghwan Kim and Robert Mackay, Simple resonance regions of torus diffeomorphisms

Willard Miller, Jr., Lecture notes in radar/sonar: Topics in Harmonic analysis with applications to radar and sonar

Calvin H. Wilcox, Lecture notes in radar/sonar: Sonar and Radar Echo Structure

Richard E. Blahut, Lecture notes in radar/sonar: Theory of remote surveillance algorithms

D.V. Anosov, Hilbert's 21st problem (according to Bolibruch)

Stephane Laederich, Ray-Singer torsion for complex manifolds and the adiabatic limit

Geneviève Raugel and George R. Sell, Navier-Stokes equations in thin 3d domains: Global regularity of solutions I

Emanuel Parzen, Time series, statistics, and information

Andrew Majda and Kevin Lamb, Simplified equations for low Mach number combustion with strong heat release

Ju. S. Il'yashenko, Global analysis of the phase portrait for the Kuramoto-Sivashinsky equation

James F. Reineck, Continuation to gradient flows

Mohamed Sami Elbialy, Simultaneous binary collisions in the collinear N-body problem

John A. Jacquez and Carl P. Simon, Aids: The epidemiological significance of two different mean rates of partner-change

Carl P. Simon and John A. Jacquez, Reproduction numbers and the stability of equilibria of SI models for heterogeneous populations

Matthew Stafford, Markov partitions for expanding maps of the circle

Ciprian Foias and Edriss S. Titi, Determining nodes, finite difference schemes and inertial manifolds

M.W. Smiley, Global attractors and approximate inertial manifolds for abstract dissipative equations

M.W. Smiley, On the existence of smooth breathers for nonlinear wave equations

Hitay Özbay and Janos Turi, Robust stabilization of systems governed by singular integro-differential equations

Mary Silber and Edgar Knobloch, Hopf bifurcation on a square lattice

Christophe Golé, Ghost circles for twist maps

Christophe Golé, Ghost tori for monotone maps

Christophe Golé, Monotone maps of $T^{n} \times R^{n}$ and their periodic orbits

E.G. Kalnins and W. Miller, Jr., Hypergeometric expansions of Heun polynomials

Victor A. Pliss and George R. Sell, Perturbations of attractors of differential equations

Avner Friedman and Peter Knabner, A transport model with micro- and macro-structure

E.G. Kalnins and W. Miller, Jr., A note on group contractions and radar ambiguity functions

George R. Sell, References on dynamical systems

Shui-Nee Chow, Kening Lu and George R. Sell, Smoothness of inertial manifolds

Shui-Nee Chow, Xiao-Biao Lin and Kening Lu, Smooth invariant foliations in infinite dimensional spaces

Kening Lu, A Hartman-Grobman theorem for scalar reaction-diffusion equations 
Christophe Golé and Glen R. Hall, Poincaré's proof of Poincaré's last geometric theorem

Mario Taboada, Approximate inertial manifolds for parabolic evolutionary equations via Yosida approximations

Peter Rejto and Mario Taboada, Weighted resolvent estimates for Volterra operators on unbounded intervals

Joel D. Avrin, Some examples of temperature bounds and concentration decay for a model of solid fuel combustion

691 Susan Friedlander and Misha M. Vishik, Lax pair formulation for the Euler equation

H. Scott Dumas, Ergodization rates for linear flow on the torus

A. Eden, A.J. Milani and B. Nicolaenko, Finite dimensional exponential attractors for semilinear wave equations with damping

A. Eden, C. Foias, B. Nicolaenko \& R. Temam, Inertial sets for dissipative evolution equations

A. Eden, C. Foias, B. Nicolaenko \& R. Temam, Hölder continuity for the inverse of Mañé's projection

697 Huanan Yang, Nonlinear wave analysis and convergence of MUSCL schemes

698 László Gerencsér and Zsuzsanna Vágó, A strong approximation theorem for estimator processes in continuous time

László Gerencsér, Multiple integrals with respect to $L$-mixing processes

David Kinderlehrer and Pablo Pedregal, Weak convergence of integrands and the Young measure representation

Bo Deng, Symbolic dynamics for chaotic systems

703 Charles Collins and Mitchell Luskin, Optimal order error estimates for the finite element approximation of the solution of a nonconvex variational problem

704 Peter Gritzmann and Victor Klee, Computational complexity of inner and outer j-radii of polytopes in finite-dimensional normed spaces

A. Ronald Gallant and George Tauchen, A nonparametric approach to nonlinear time series analysis: estimation and simulation

H.S. Dumas, J.A. Ellison and A.W. Sáenz, Axial channeling in perfect crystals, the continuum model and the method of averaging

M.A. Kaashoek and S.M. Verduyn Lunel, Characteristic matrices and spectral properties of evolutionary systems

Xinfu Chen, Generation and Propagation of interfaces in reaction diffusion systems

Avner Friedman and Bei Hu, Homogenization approach to light scattering from polymer-dispersed liquid crystal films

Yoshihisa Morita and Shuichi Jimbo, ODEs on inertial manifolds for reaction-diffusion systems in a singularly perturbed domain with several thin channels

Wenxiong Liu, Blow-up behavior for semilinear heat equations:multi-dimensional case

Hi Jun Choe, Hölder continuity for solutions of certain degenerate parabolic systems

Hi Jun Choe, Regularity for certain degenerate elliptic double obstacle problems

Fernando Reitich, On the slow motion of the interface of layered solutions to the scalar Ginzburg-Landau equation

Xinfu Chen and Fernando Reitich, Local existence and uniqueness of solutions of the Stefan problem with surface tension and kinetic undercooling rings

W. Balser, B.L.J. Braaksma, J.-P. Ramis and Y. Sibuya, Multisummability of formal power series solutions of linear ordinary differential equations

Peter J. Olver and Chehrzad Shakiban, Dissipative decomposition of partial differential equations

Clark Robinson, Homoclinic bifurcation to a transitive attractor of Lorenz type, II

Michelle Schatzman, A simple proof of convergence of the $Q R$ algorithm for normal matrices without shifts

Ian M. Anderson, Niky Kamran and Peter J. Olver, Internal, external and generalized symmetries

C. Foias and J.C. Saut, Asymptotic integration of Navier-Stokes equations with potential forces. I

Ling Ma, The convergence of semidiscrete methods for a system of reaction-diffusion equations

Adelina Georgescu, Models of asymptotic approximation

A. Makagon and H.Salehi, On bounded and harmonizable solutions on infinite order arma systems

San-Yih Lin and Yan-Shin Chin, An upwind finite-volume scheme with a triangular mesh for conservation laws

J.M. Ball, P.J. Holmes, R.D. James, R.L. Pego \& P.J. Swart, On the dynamics of fine structure

KangPing Chen and Daniel D. Joseph, Lubrication theory and long waves

J.L. Ericksen, Local bifurcation theory for thermoelastic Bravais lattices 